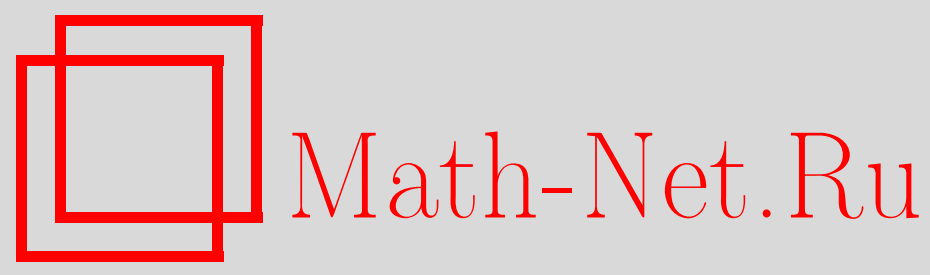

B. Е. Тараканов, Новые серии рациональных аппроксимаций и некоторые их применения, Матем. заметки, 2004, том 76, выпуск 2, 237-257

DOI: https://doi.org/10.4213/mzm103

Использование Общероссийского математического портала Math-Net.Ru подразумевает, что вы прочитали и согласны с пользовательским соглашением http://www . mathnet.ru/rus/agreement

Параметры загрузки:

IP : 54.205 .225 .156

26 апреля 2023 г., 10:42:14

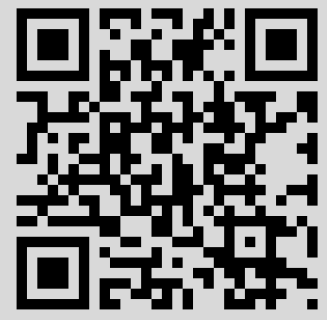




\title{
НОВЫЕ СЕРИИ РАЦИОНАЛЬНЫХ АППРОКСИМАЦИЙ И НЕКОТОРЫЕ ИХ ПРИМЕНЕНИЯ
}

\author{
В. Е. Тараканов
}

Рассматривается известная задача дискретного логарифмирования в конечном простом поле $G F(p), p$ - простое число, имеющая ряд приложений в вопросах защиты информации. В п. 1 вводятся и изучаются некоторые числовые последовательности, возникающие при разложении вещественного числа в цепную дробь. Полученные результаты применяются в п. 2, где вводится новый алгоритм, основанный на рациональных аппроксимациях, для решения задачи представления дискретного логарифма данного числа в виде суммы логарифмов малых простых чисел; эта задача является важнейшей составной частью задачи дискретного логарифмирования. Получен ряд результатов, необходимых для построения и обоснования алгоритма представления. Точная формулировка этого алгоритма дается в п. 3. Приводятся некоторые экспериментальные результаты, иллюстрирующие работу алгоритма в случае простых чисел порядков $10^{16}-10^{31}$.

Библиограффия: 6 названий.

Используемые в работе теоретико-числовые понятия см. в книге [1], терминологию и результаты, касающиеся рациональной апшроксимации, см. в книге [2].

Пусть $p$ - заданное простое, $v$ - любое целое число, $0<v<p, t$ - представитель смежного класса, являющегося образующим элементом мультипликативной грушы конечного простого поля $G F(p)$. Дискретным логарифмом (или индексом) числа $v$ по модулю $p$ назьвается наименьшее целое положительное число $\lg v$ со свойством

$$
t^{\lg v} \equiv v(\bmod p) .
$$

Нахождение эффективного способа вычисления $\lg v$ для больших (с несколькими десятками десятичных знаков) чисел $p$ является весьма трудной задачей. Она имеет немаловажное практическое значение, так как на ее большой вычислительной сложности основан цельй рад известных систем защиты информации (см. [3]-[5]). Наиболее распространенные современные алгоритмы основаны на сложных алгебраических и теоретико-числовых конструкциях и включают такие составные части, как разложение на множители больших целых чисел и вероятностные алгоритмы поиска эллиптических кривьх над $G F(p)$ с порядком, делящимся лишш на произведения малых простых чисел (см. [5]-[6]). Все они представляют собой вероятностные алгоритмы и имеют субэкспоненциальную по $\log p$ сложность.

Работа выполнена при частичной поддержке государственной программы "Ведущие научные школы", грант НШ-1758.2003.1. 
Мы представляем здесь алгоритм для решения важнейшей составной части задачи дискретного логарифмирования - задачи нахождения представлений $\lg v$ в виде суммы дискретных логарифмов малых простых чисел. В отличие от упомянутых вьше алгоритмов он основан лишь на теории рациональной аппроксимации вещественных чисел. Алгоритм также носит вероятностный характер, он имеет весьма простую логическую структуру. Мы полагаем, что представленньй алгоритм может послужить основой для построения эффективного вероятностного алгоритма дискретного логарифмирования.

В п. 1 мы вводим и изучаем некоторые новые объекты, связанные с разложениями вещественных чисел в цепную дробь. В п. 2 приводится ряд результатов, необходимых для построения и, вместе с результатами п. 1, обоснования алгоритма представления $\lg v$ в виде суммы логарифмов малых простых чисел. В п. 3 мы формулируем этот алгоритм. Приводятся также иллюстрации действий по нему и некоторые результаты проведенных численных экспериментов для простых чисел $p$ порядков $10^{15}-10^{30}$.

1. Свойства цепных дробей. В наших рассмотрениях будем опираться на книгу [2]. Пусть $\gamma$ - вещественное число, $0<\gamma<1$; если $\gamma$ рационально, то оно берется в своем несократимом представлении. Для $\gamma$ строятся две последовательности: $a_{0}=0, a_{1}, a_{2}, \ldots$ и $\gamma_{0}=\gamma^{-1}, \gamma_{1}, \gamma_{2}, \ldots$ :

$$
a_{i}=\left[\gamma_{i-1}^{-1}\right], \quad \gamma_{i}=\gamma_{i-1}^{-1}-a_{i}, \quad i=1,2, \ldots
$$

Члены последовательности $a_{0}, a_{1}, a_{2}, \ldots$ называются әлементами цепной дроби, изображающей $\gamma$. Далее рассматривается последовательность $\left\{k_{i} / e_{i}\right\}, i=0,1,2, \ldots$ :

$$
\begin{aligned}
& k_{0}=0, \quad k_{1}=1, \quad e_{0}=1, \quad e_{1}=a_{1}, \\
& k_{n}=a_{n} k_{n-1}+k_{n-2}, \\
& e_{n}=a_{n} e_{n-1}+e_{n-2}, \quad n \geqslant 2 .
\end{aligned}
$$

Получающиеся согласно (1)-(3) несократимые дроби $k_{n} / e_{n}, n=0,1,2, \ldots$ дают наилучшие приближения $\gamma$ рациональными числами, т.е. для любого $n$ справедливо $\left|k_{n} / e_{n}-\gamma\right|<|r / s-\gamma|$, если $s \leqslant e_{n}, r / s \neq k_{n} / e_{n}$. Дробь $k_{n} / e_{n}$ назьвается $n$-й подходящей дробъю для $\gamma$, а нахождение последовательности $a_{0}, a_{1}, a_{2}, \ldots-$ разложсением $\gamma$ в цепную дробь. Справедливы следующие утверждения.

1) Для $n \geqslant 2$ вьполнено

$$
e_{n} k_{n-1}-k_{n} e_{n-1}=(-1)^{n} \text {. }
$$

2) Для $n \geqslant 2$ вьполнено

$$
e_{n} k_{n-2}-k_{n} e_{n-2}=(-1)^{n-1} a_{n}
$$

3) Последовательность подходящих дробей для $\gamma$ конечна тогда и только тогда, когда $\gamma$ рационально; в этом случае ее последний член совпадает с $\gamma$.

4) Для любого четного $n \geqslant 2$ и целого $r \geqslant 1$

$$
\frac{k_{n-2 r}}{e_{n-2 r}}<\cdots<\frac{k_{n-2}}{e_{n-2}} \leqslant \gamma \leqslant \frac{k_{n-1}}{e_{n-1}}<\frac{k_{n-3}}{e_{n-3}}<\cdots<\frac{k_{n-2 r-1}}{e_{n-2 r-1}}
$$


равенство в (6) достигается лиш при рациональном $\gamma$ - для последней подходящей дроби (см. (3)).

Напомним еще два общих закона рациональной аппроксимации вешественных чисел, установленных с помощью теории цепных дробей.

I. Для любого иррационального числа $\sigma$ и любого вещественного числа $\tau$ неравенство $|\sigma x-y-\tau|<3 / x$ имеет бесконечное число решений в целых числах $x$ и $y, x>0$.

II. Для того чтобы существовало положительное число $C$, обладающее тем свойством, что при любых вещественных $n \geqslant 1$ и $\tau$ найдется пара целых чисел $x$ и $y, x>0$, удовлетворяющих неравенствам

$$
x \leqslant C n, \quad|\sigma x-y-\tau|<\frac{1}{n},
$$

необходимо и достаточно, чтобы $\sigma$ представлялось цепной дробью с ограниченньми элементами.

ЗАмЕЧАниЕ. ЭТи общие законы аппроксимации сформулированы для иррациональных чисел $\sigma$. Однако они при надлежащей интерпретации имеют отношение и к рассматриваемьм в работе рациональньм числам $\gamma, 0<\gamma<1$, если те имеют большое число знаков в десятичном разложении, так как такие рациональные $\gamma$ можно фактически задать лишь приближенно, с достаточной для наших целей степенью точности, а это приближенное значение $\tilde{\gamma} \approx \gamma$ с не меньшей степенью точности апшроксимируется бесконечными цепными дробями с ограниченными элементами.

Наряду с подходящими дробями в теории цепных дробей рассматриваются также промежуточные дроби, определяемые для всех $n \geqslant 2$ при $a_{n}>1$. Для $n \geqslant 2$ рассмотрим последовательность $R_{n}$ :

$$
R_{n}(i)=\frac{k_{n-2}+i k_{n-1}}{e_{n-2}+i e_{n-1}}, \quad i=0,1, \ldots, a_{n} .
$$

Ясно, что $R_{n}(0)=k_{n-2} / e_{n-2}, R_{n}\left(a_{n}\right)=k_{n} / e_{n}$ (см. $\left.(2),(3)\right)$, т.е. в $R_{n}$ входят $(n-2)$-я и $n$-я подходяшие дроби. Члены (7) с $i=1,2, \ldots, a_{n}-1$ назьваются $n$-ми промежуточны.ми дробями. Из общей теории аппроксимации известно, что любое наилучшее приближение вещественного числа есть либо подходящая, либо промежуточная дробь. Из (7) непосредственно выводится, что

$$
\begin{aligned}
& \frac{k_{n-2}}{e_{n-2}}<R_{n}(i)<\frac{k_{n}}{e_{n}}, \text { если } n \text { четно, } \\
& \frac{k_{n-2}}{e_{n-2}}>R_{n}(i)>\frac{k_{n}}{e_{n}}, \text { если } n \text { нечетно, }
\end{aligned}
$$

Мы введем теперь обобщенные промежуточные дроби как некоторые бесконечные последовательности положительных рациональных дробей. Они, вообще говоря, не дают наилучших приближений, однако, как мы покажем, позволяют получать бесконечно много приближений заданной точности для любого вещественного числа $\sigma$. 
Пусть $s>0, t$ - целые числа, НОД $(s, t)=1, n \geqslant 2$ - заданное целое число, $k_{n-2} / e_{n-2}$, $k_{n-1} / e_{n-1}, k_{n} / e_{n}-$ подходящие дроби для данного вещественного числа $\gamma$. Рассмотрим две последовательности $R_{n}^{(s, t)}=\left(R_{n}^{(s, t)}(i)\right), R_{n}^{(s, t)}=\left(R_{n}^{(s, t)}(i)\right), i=1,2, \ldots$, где

$$
\begin{aligned}
R_{n}^{(s, t)}(i)=\frac{i s k_{n-1}+t k_{n-2}}{i s e_{n-1}+t e_{n-2}}, & i=1,2, \ldots \\
R_{n}^{(s, t)}(i)=\frac{s k_{n-1}+i t k_{n-2}}{s e_{n-1}+i e_{n-2}}, & i=1,2, \ldots
\end{aligned}
$$

Положительные члены последовательностей $R_{n}^{(s, t)}, R_{n}^{(s, t)}$ назовем $n$-мu $(s, t)$-npoмeжуточными дробями для $\gamma$.

Установим ряд свойств последовательностей $R_{n}^{(s, t)}, R_{n}^{(s, t)}$. Если $t>0$, то все их члены положительны, следовательно, представляют собой $n$-е $(s, t)$-промежуточные дроби.

ПРЕДЛОЖЕНИЕ 1. Пусть $t<0$.

а) Члены последовательности $R_{n}^{(s, t)}$ положительны всюду, кроме значений $i$, принадлежсащих интервалу

$$
\left(\frac{|t|}{s} \frac{k_{n-2}}{k_{n-1}}, \frac{|t|}{s} \frac{e_{n-2}}{e_{n-1}}\right)
$$

при четном $n$ и интервалу

$$
\left(\frac{|t|}{s} \frac{e_{n-2}}{e_{n-1}}, \frac{|t|}{s} \frac{k_{n-2}}{k_{n-1}}\right)
$$

при п нечетном.

б) Члены последовательности $R_{n}^{(s, t)}$ положительны всюду, кроме тех значений $i$, которые принадлежсат интервалу

$$
\left(\frac{s}{|t|} \frac{e_{n-1}}{e_{n-2}}, \frac{s}{|t|} \frac{k_{n-1}}{k_{n-2}}\right)
$$

при четном $n$ и интервалу

$$
\left(\frac{s}{|t|} \frac{k_{n-1}}{k_{n-2}}, \frac{s}{|t|} \frac{e_{n-1}}{e_{n-2}}\right)
$$

при п нечетном.

ДокАЗАтельство. а) Рассмотрим $R_{n}^{(s, t)}$. Пусть $n$ четно. Тогда ввиду (6)

$$
\frac{k_{n-2}}{e_{n-2}}<\frac{k_{n-1}}{e_{n-1}}
$$

и, следовательно,

$$
\frac{k_{n-2}}{k_{n-1}}<\frac{e_{n-2}}{e_{n-1}}, \quad \text { если } n \text { четно. }
$$


Аналогично, если $n$ нечетно, то

$$
\frac{k_{n-1}}{e_{n-1}}<\frac{k_{n-2}}{e_{n-2}}
$$

и получаем

$$
\frac{e_{n-2}}{e_{n-1}}<\frac{k_{n-2}}{k_{n-1}}, \quad \text { если } n \text { нечетно. }
$$

Из (10)-(11) вытекает, что

$$
-\frac{t}{s} \frac{k_{n-2}}{k_{n-1}}=\frac{|t|}{s} \frac{k_{n-2}}{k_{n-1}}<\frac{|t|}{s} \frac{e_{n-2}}{e_{n-1}}
$$

при четном $n$. Аналогично, из (12)

$$
\frac{|t|}{s} \frac{e_{n-2}}{e_{n-1}}<\frac{|t|}{s} \frac{k_{n-2}}{k_{n-1}}
$$

при $n$ нечетном. Из (8) следует, что $R_{n}^{(s, t)}(i)$ может быть отрищательньм для $i>0$ только, если $i$ попадает в интервал

$$
\left(\frac{|t|}{s} \frac{k_{n-2}}{k_{n-1}}, \frac{|t|}{s} \frac{e_{n-2}}{e_{n-1}}\right)
$$

при $n$ четном, и в

$$
\left(\frac{|t|}{s} \frac{e_{n-2}}{e_{n-1}}, \frac{|t|}{s} \frac{k_{n-2}}{k_{n-1}}\right)
$$

при $n$ нечетном.

б) Рассмотрим $R_{n}^{(s, t)}$. Из (6) получаем

$$
\begin{aligned}
& \frac{k_{n-1}}{k_{n-2}}>\frac{e_{n-1}}{e_{n-2}}, \quad n \text { четно, } \\
& \frac{k_{n-1}}{k_{n-2}}<\frac{e_{n-1}}{e_{n-2}}, \quad n \text { нечетно. }
\end{aligned}
$$

Из (9) и (13) следует теперь искомьй результат.

ПРЕДЛОЖЕНИЕ 2. Пусть $n \geqslant 2$. Если $t>0$, то последовательность $R_{n}^{(s, t)} n p u$ четном $n$ монотонно возрастает, при нечетном $n$ монотонно убывает.

Пусть $t<0$. Тогда при четном $n R_{n}^{(s, t)}$ монотонно убывает всюду, кроме значения $\left[(|t| / s)\left(e_{n-2} / e_{n-1}\right)\right]$ (если оно положстельно). При нечетном $n R_{n}^{(s, t)}$ монотонно возрастает всюду, кроме значения $\left[(|t| / s)\left(e_{n-2} / e_{n-1}\right)\right]($ если оно положительно).

ДокАЗАТЕЛЬСтво. Учитывая свойство 1) (см. выше), имеем

$$
R_{n}^{(s, t)}(i+1)-R_{n}^{(s, t)}(i)=\frac{(-1)^{n} s t}{\left((i+1) s e_{n-1}+t e_{n-2}\right)\left(i s e_{n-1}+t e_{n-2}\right)} .
$$

Из этого равенства следуют все требуемые утверждения. 
ПРЕДЛОЖЕНИЕ $2^{\prime}$. Пусть $n \geqslant 2$. Если $t>0$, то последовательность $R_{n}^{\prime(s, t)}$ монотонно убавает при четном $n$ и монотонно возрастает при $n$ нечетном.

Пусть $t<0$. Тогда при четном $n R_{n}^{(s, t)}$ монотонно возрастает всюду, кроме значения

$$
i=\left[\frac{s}{|t|} \frac{e_{n-1}}{e_{n-2}}\right]
$$

(если оно положительно). При нечетном $n R_{n}^{(s, t)}$ монотонно убывает всюду, кроме значения

$$
i=\left[\frac{s}{|t|} \frac{e_{n-1}}{e_{n-2}}\right]
$$

(если оно положительно).

Это доказывается аналогично предложению 2.

ПРЕДЛОЖЕНИЕ 3. Для последовательности $R_{n}^{(s, t)}$ справедливо

a) $\lim _{i \rightarrow \infty} R_{n}^{(s, t)}(i)=k_{n-1} / e_{n-1}$;

б) при четном $n R_{n}^{(s, t)}(i)>k_{n-2} / e_{n-2}\left(\right.$ при $t>0$ идля $i>(|t| / s)\left(e_{n-2} / e_{n-1}\right)$ npu $t<0)$;

в) при нечетном $n R_{n}^{(s, t)}(i)<k_{n-2} / e_{n-2}($ при тех жсе условия $x)$.

ДоКАЗАТЕЛЬСТво. Учитьвая свойство 1) (см. выше), имеем

$$
\frac{k_{n-1}}{e_{n-1}}-R_{n}^{(s, t)}(i)=\frac{(-1)^{n} t}{e_{n-1}\left(i s e_{n-1}+t e_{n-2}\right)} .
$$

При

$$
i>\frac{|t|}{s} \frac{e_{n-2}}{e_{n-1}}
$$

все величины в правой части имеют одинаковые знаки. По предложению $2 R_{n}^{(s, t)}$ монотонны, и те из них, которые возрастают (т.е. когда $t>0, n$ четно или $t<0, n$ нечетно) ограничены сверху, а те, что монотонно убывают (когда $t<0, n$ четно или $t>0, n$ нечетно) ограничены снизу одной и той же величиной $k_{n-1} / e_{n-1}$. Предел $\lim _{i \rightarrow \infty} R_{n}^{(s, t)}(i)$ существует и равен $k_{n-1} / e_{n-1}$. Далее, аналогично предыдущему, легко увидеть, что

$$
R_{n}^{(s, t)}(i)-\frac{k_{n-2}}{e_{n-2}}=\frac{(-1)^{n} i s}{e_{n-2}\left(i s e_{n-1}+t e_{n-2}\right)}
$$

Из этого равенства следуют утверждения б) и в). Предложение доказано.

ПРЕДЛОЖЕНИЕ $3^{\prime}$. Для последовательности $R_{n}^{(s, t)}$ справедливо:

а) $\lim _{i \rightarrow \infty} R_{n}^{(s, t)}=k_{n-2} / e_{n-2}$;

б) при четном $n R_{n}^{(s, t)}(i)>k_{n-2} / e_{n-2}$ nрu $t>0 u<k_{n-2} / e_{n-2}$ для $i>(s /|t|) \times$ $\left(e_{n-1} / e_{n-2}\right)$ npu $t<0$

в) при $n$ нечетном $R_{n}^{(s, t)}(i)<k_{n-2} / e_{n-2} n p u t>0 u>k_{n-2} / e_{n-2}$ для $i>(s /|t|)\left(e_{n-1} / e_{n-2}\right)$ npu $t<0$. 
Это доказывается аналогично предложению 3.

Предложения 3 и $3^{\prime}$ дают практический способ, имея всего лишь два последовательных наилучших приближения к данному числу $\gamma$, строить непосредственно по ним сколь угодно много приближений примерно той же точности, среди которых заведомо найдутся приближения более точные. Последнее замечание можно конкретизировать. Напомним, что если $k_{n-2} / e_{n-2}, k_{n-1} / e_{n-1}, k_{n} / e_{n}$ - подходящие дроби для числа $\gamma$, то (см. свойство 4))

$$
\begin{aligned}
& \frac{k_{n-2}}{e_{n-2}}<\frac{k_{n}}{e_{n}}<\gamma<\frac{k_{n-1}}{e_{n-1}}, \quad \text { если } n \text { четно, } \\
& \frac{k_{n-1}}{e_{n-1}}<\gamma<\frac{k_{n}}{e_{n}}<\frac{k_{n-2}}{e_{n-2}}, \quad \text { если } n \text { нечетно. }
\end{aligned}
$$

С учетом (14) формулируем следуюший результат.

ПРЕДЛОЖЕНИЕ 4. Пусть $n \geqslant 2$ - натуральное число, $k_{n-2} / e_{n-2}, k_{n-1} / e_{n-1}$, $k_{n} / e_{n}$ - подходящие дроби для данного числа $\gamma, 0<\gamma<1$. Для любих иелых $s, t>0$ в последовательности $R_{n}^{(s, t)}$ содержится бесконечно много членов c $R_{n}^{(s, t)}(i) \in\left(k_{n} / e_{n}, k_{n-1} / e_{n-1}\right)$ при четном $n$ и с $R_{n}^{(s, t)}(i) \in\left(k_{n-1} / e_{n-1}, k_{n} / e_{n}\right)$ при $n$ нечетном, а именно, все члены $R_{n}^{(s, t)}(i)$ с $i>(t / s) a_{n}$, әде $a_{n}-n$-й әлемент разложсения $\gamma$ в иепную дробь. В последовательности $R_{n}^{(s, t)}$ cs $s 0, t<0$ таких членов нет.

ДоКАЗАТЕЛЬСТВО. Согласно свойствам 1) и 2) имеем

$$
R_{n}^{(s, t)}(i)-\frac{k_{n}}{e_{n}}=\frac{(-1)^{n} i s+(-1)^{n-1} a_{n} t}{e_{n}\left(i s e_{n-1}+t e_{n-2}\right)}
$$

где $a_{n}-n$-й элемент разложения $\gamma$ в цепную дробь. Пусть сначала $n$ четно. Тогда для $t>0$ в силу предложений 2 и 3

$$
R_{n}^{(s, t)}(i)<\frac{k_{n-1}}{e_{n-1}}, \quad i>0 .
$$

Ввиду (15) условие $R_{n}^{(s, t)}(i)>k_{n} / e_{n}$ равносильно

$$
i>\frac{t}{s} a_{n}
$$

Для $t<0$ ввиду $(15) R_{n}^{(s, t)}(i)>k_{n} / e_{n}$ равносильно

$$
i>\frac{|t|}{s} \frac{e_{n-2}}{e_{n-1}} .
$$

Но для этих значений $i$ согласно предложениям 2 и 3

$$
R_{n}^{(s, t)}(i)>\frac{k_{n-1}}{e_{n-1}}
$$

Тем самым получаем, что при $t<0$ в $\left(k_{n} / e_{n}, k_{n-1} / e_{n-1}\right)$ чисел из $R_{n}^{(s, t)}$ нет. 
Пусть теперь $n$ нечетно. Тогда для $t>0$ вьполняется

$$
R_{n}^{(s, t)}(i)>\frac{k_{n-1}}{e_{n-1}}, \quad i>0
$$

Так как при нечетном $n$ (см. (15))

$$
R_{n}^{(s, t)}(i)-\frac{k_{n}}{e_{n}}=\frac{-i s+a_{n} t}{e_{n}\left(i s e_{n-1}+t e_{n-2}\right)},
$$

условие $R_{n}^{(s, t)}(i)<k_{n} / e_{n}$ равносильно (16). Для $t<0$, аналогично предыдущему, убеждаемся, что $R_{n}^{(s, t)}(i)<k_{n} / e_{n}$ лишь тогда, когда $R_{n}^{(s, t)}(i)<k_{n-1} / e_{n-1}$. Предложение доказано.

ПРЕДЛОЖЕНИЕ $4^{\prime}$. Пусть $n \geqslant 2$ - натуральное число, $k_{n-2} / e_{n-2}, k_{n-1} / e_{n-1}$, $k_{n} / e_{n}-$ подходящие дроби для данного числа $\gamma, a_{n}-n$-й әлемент разложения $\gamma$ в чепную дробь. Для любого иелого $s>a_{n} u$ любого иелого $t$, для которого $0<t<s / a_{n}$, в $R_{n}^{(s, t)}$ найдутся члены такие, что

$$
\begin{array}{ll}
R_{n}^{(s, t)}(i) \in\left(\frac{k_{n}}{e_{n}}, \frac{k_{n-1}}{e_{n-1}}\right) & \text { при четном } n, \\
R_{n}^{(s, t)}(i) \in\left(\frac{k_{n-1}}{e_{n-1}}, \frac{k_{n}}{e_{n}}\right) & \text { при нечетном } n,
\end{array}
$$

а именно, члены $R_{n}^{(s, t)}(i)$ для всех $i \in\left[1, s /\left(a_{n} t\right)\right)$. При $t \geqslant s / a_{n} u t<0$ таких членов в $R_{n}^{\prime(s, t)}$ нет.

ДоКАЗАТЕЛЬСТВО. Пользуясь свойствами 1), 2), находим

$$
R_{n}^{(s, t)}(i)-\frac{k_{n}}{e_{n}}=\frac{(-1)^{n} s+(-1)^{n-1} i a_{n} t}{e_{n}\left(s e_{n-1}+i t e_{n-2}\right)}
$$

Находим также

$$
\frac{k_{n-1}}{e_{n-1}}-R_{n}^{(s, t)}(i)=\frac{(-1)^{n} i t}{e_{n-1}\left(s e_{n-1}+i t e_{n-2}\right)} .
$$

Пусть сначала $n$ четно. Тогда из (18) при $t>0$ следует, что

$$
R_{n}^{(s, t)}(i)<\frac{k_{n-1}}{e_{n-1}}, \quad i>0 .
$$

Далее, из (17) ввиду монотонности $R_{n}^{(s, t)}$, вытекает, что при $t>0 k_{n} / e_{n}<R_{n}^{(s, t)}(i)$ лишь для $i \in\left[1, s /\left(a_{n} t\right)\right)$. Этот интервал может содержать целые числа лишь при выполнении условия $t<s / a_{n}$.

При $t<0$ по $(18)$ имеем $R_{n}^{\prime(s, t)}(i)>k_{n-1} / e_{n-1}$, когда $i<(s /|t|)\left(e_{n-1} / e_{n-2}\right)$, и $R_{n}^{(s, t)}(i)<k_{n-1} / e_{n-1}$ в противном случае. Однако в этом последнем случае, как показано выше,

$$
R_{n}^{(s, t)}<\frac{k_{n-2}}{e_{n-2}}<\frac{k_{n}}{e_{n}} .
$$


Таким образом, при $n$ четном и $t$ отрицательном членов последовательности $R_{n}^{\prime(s, t)}$ в $\left(k_{n} / e_{n}, k_{n-1} / e_{n-1}\right)$ нет.

Для $n$ нечетного, рассуждая совершенно аналогично, также приходим к искомому результату. Предложение доказано.

Установленные в предложениях $3,3^{\prime}$ и $4,4^{\prime}$ свойства обобщенных промежуточных дробей мы применим в п. 2 к обоснованию одного алгоритма дискретного логарифмирования в конечном простом поле.

2. Представление дискретного логарифма целого числа в виде суммы логарифмов малых простых чисел. Пусть $p$ - заданное простое, $v$ - целое число, $0<v<p$. Пусть $\mathscr{B}=\{2,3, \ldots\}$ - конечное множество, состояшее из всех наименьших простых чисел, $|\mathscr{B}|$ - его мощность. Целое число, делящееся лиш на произведения чисел из $\mathscr{B}$, назовем гладким. Будем называть малым целое число, абсолютная величина которого принадлежит отрезку натурального ряда с достаточно большой плотностью гладких чисел; это определение получает точньй смысл, как только мы указьваем множество $\mathscr{B}$ и конкретное значение плотности. Мы будем стремиться получать соотношения вида

$$
s_{1} v \equiv s_{2}(\bmod p)
$$

с возможно более малыми $s_{1}, s_{2}$, исходя из которых будем находить соотношения (19) с гладкими $s_{1}$ и $s_{2}$. Это и даст решение задачи о представлении.

Сначала приводим задачу к случаю

$$
v \in I, \quad \text { где } \quad I=\left(\frac{3}{8} p, \frac{3}{7} p\right) .
$$

Назовем целые числа $v_{1}$ и $v_{2}$ әквивалентны.мu, $v_{1} \sim v_{2}$, если мы можем эффективно определить такие гладкие числа $s^{\prime}$ и $s^{\prime \prime}$, что

$$
s^{\prime} v_{1} \equiv s^{\prime \prime} v_{2}(\bmod p)
$$

ПРЕДЛОЖЕНИЕ 5. Для любого иелого $v, 0<v<p$, не более, чем за $O(\log p)$ операций, мохно найти такое целое число $v^{\prime}$, что $v^{\prime} \sim v$ u $v^{\prime} \in I$. Под операцией мы понимаем здесь умножсение на малое (не больше 12) челое число с последующим приведением по модулю р.

Доказательства этого и последующих предложений 6-8 достаточно элементарны, хотя иногда и довольно громоздки. Поэтому в данной работе мы их опускаем.

Ввиду предложения 5 мы в дальнейшем ограничиваемся случаем $v \in I$.

Определим рациональное число $\alpha=\alpha(v)$ :

$$
\alpha=\frac{p-2 v}{v} .
$$

Имеем

$$
v(2+\alpha)=p .
$$

Ясно, что $\alpha(v)$ убьвает в интервале $I$ от $2 / 3$ до $1 / 3$. 
Если $\alpha$ в (20), (21) заменить какой-либо рациональной дробью $\tilde{\alpha}$, достаточно мало отличающейся от $\alpha$, и записать $2+\tilde{\alpha}=l_{1} / l_{2}, l_{1}, l_{2}$ целье, НОД $\left(l_{1}, l_{2}\right)=1$, то

$$
l_{1} v \equiv d(\bmod p)
$$

с достаточно малым $d=l_{1} v-l_{2} p$. Разобьем $I$ на два интервала $I_{1}$ и $I_{2}$ :

$$
I_{1}=\left(\frac{3}{8} p, \frac{2}{5} p\right], \quad I_{2}=\left(\frac{2}{5} p, \frac{3}{7} p\right)
$$

для $v \in I_{1}$ имеем оценку $2 / 3>\alpha>1 / 2$, для $v \in I_{2}$ - оценку $1 / 2>\alpha>1 / 3$. В целях большей прозрачности изложения ограничимся далее лишь случаем $v \in I_{1}$. Подчеркнем, что для $v \in I_{2}$ все последующие рассуждения вполне аналогичны, с изменением, главным образом, коэффициентов в ряде формул.

Итак, далее пусть

$$
v \in\left(\frac{3}{8} p, \frac{2}{5} p\right), \quad \frac{2}{3}>\alpha(v)>\frac{1}{2} .
$$

Пусть $x, e-$ два цельх числа, $x>|e|>0$. Введем дробно-линейные функции от $x, e$ :

$$
\alpha(x, e)= \begin{cases}\frac{x+e}{2 x+e}, & e>0 \\ \frac{2 x+e}{3 x+e}, & e<0\end{cases}
$$

а также линейные функции

$$
l_{1}(x, e)= \begin{cases}5 x+3 e, & e>0 \\ 8 x+3 e, & e<0\end{cases}
$$

и

$$
l_{2}(x, e)= \begin{cases}2 x+e, & e>0, \\ 3 x+e, & e<0 .\end{cases}
$$

Ясно, что для функций из (22)-(24) вьполняется равенство

$$
2+\alpha(x, e)=\frac{l_{1}(x, e)}{l_{2}(x, e)} .
$$

Мы будем заменять в $(21)$ число $\alpha$ значениями $\alpha(x, e)$ из $(22)$. Такую замену при конкретных целочисленных значениях $x, e, x>|e|>0$, назовем $(x, e)$-заменой: $\alpha \rightarrow \alpha(x, e)$. Скажем, что замена $\alpha \rightarrow \alpha(x, e)$ приводит к соотношению

$$
l_{1}(x, e) v \equiv d(x, e)(\bmod p),
$$

если

$$
d(x, e)=l_{1}(x, e) v-l_{2}(x, e) p
$$

и $l_{1}(x, e), l_{2}(x, e)$ - функции из $(23),(24)$. Мы дадим ниже способ получения с использованием описанных вьше $(x, e)$-замен соотношений вида $(19)$ с гладкими $s_{1}, s_{2}$. При этом производим $(x, e)$-замены, непременно соблюдая сформулированное ниже правило С. Разобьем $I_{1}$ на два интервала:

$$
I_{1}=I_{1}^{\prime} \cup I_{1}^{\prime \prime}, \quad \text { где } I_{1}^{\prime}=\left(\frac{3}{8} p, \frac{5}{13} p\right], \quad I_{1}^{\prime \prime}=\left(\frac{5}{13} p, \frac{2}{5} p\right],
$$


ПрАвило С (правило $(x, e)$-замен). Если $v \in I_{1}^{\prime}$, то берем $e>0$ и

$$
\alpha \rightarrow \alpha(x, e)=\frac{x+e}{2 x+e}
$$

если $v \in I_{1}^{\prime \prime}$, то берем $e<0$ и

$$
\alpha \rightarrow \alpha(x, e)=\frac{2 x-|e|}{3 x-|e|} .
$$

Для оценки абсолютной величины чисел $d(x, e)$ из $(25),(26)$, к которым приходим в результате $(x, e)$-замен, вводим параметр $\beta=\beta(v)$ :

$$
\beta= \begin{cases}(1-\alpha)^{-1}-2, & v \in I_{1}^{\prime} \\ 3-(1-\alpha)^{-1}, & v \in I_{1}^{\prime \prime}\end{cases}
$$

с $\alpha$ из (20), $I_{1}^{\prime}, I_{1}^{\prime \prime}$, из (27).

Для последующего существенно свойство

$$
1<\beta^{-1}<2
$$

при любом $v \in I_{1}$.

ПРЕДЛОЖЕНИЕ 6. Пусть $v \in I_{1}$. Если мы производим $(x, e)$-замену с соблюдением правила $\mathrm{C}$, то приходим к соотношению (25) $c$

$$
|d(x, e)|=\left|\beta-\frac{|e|}{x}\right|(1-\alpha) x v,
$$

где $d(x, e)$ из (26), $\beta$ из (28).

При $(x, e)$-замене может быть обеспечено условие делимости коэффициента $l_{1}(x, e)$ в левой части (25) на произвольное целое число $q$.

Пусть

$$
l=l(v)= \begin{cases}5, & v \in I_{1}^{\prime}, \\ 8, & v \in I_{1}^{\prime \prime} .\end{cases}
$$

Через $\rho(q)$ будем обозначать наименьший неотрицательный вычет по модулю $l$ рационального числа $q$ со знаменателем, взаимно простым $c l$. Предполагая производить далее $(x, e)$-замены (с соблюдением правила $\mathrm{C})$, определяем

$$
\mu=\mu(q)=\rho\left(3 q^{-1} \operatorname{sgn} e\right)
$$

для целого $q$, взаимно простого с $v$; если $v$ фиксировано, то $l$ и sgn $e$ также фиксированы, и $\mu$ зависит только от $q$. Полагаем далее

$$
m=m(q)=\frac{\mu q-3 \operatorname{sgn} e}{l}
$$

c $l$ из (31), $\mu$ из (32). Легко увидеть, что для любого $v \in I_{1}$ и любого целого $e \neq 0$ число $m(q)$ целое и $0<m(q)<q$. 
ПРЕДЛОЖЕНИЕ 7. Пусть $v \in I_{1}$ иелое, $q>3$ - натуральное число, НОД $(q, l)=1$, где $l$ - число из (31). Тогда справедливы следующие утвержсдения.

1) Если в результате $(x, e)$-замены мы приходим $\kappa(25)$ с $q \mid l_{1}(x, e)$, то

$$
x=|e| m+k q
$$

для $m$ из (33) и некоторого иелого $k$.

2) Обратно, пусть е, $k$ - любые челье числа, е, $k \neq 0$, a $x$ вычисляется по формуле (34) с тиз (33). Если при этом $x>|e|$, то в результате $(x, e)$-замены мы приходим $\kappa(25)$ с $q \mid l_{1}(x, e)$.

Заметим, что условие $x>|e|$ в последнем утверждении выполняется, как легко убедиться, не менее, чем для половины всех возможных пар чисел $(k, e)$.

Мы укажем теперь способ получения для данного целого $v \in I$ и какого-либо целого $q$, НОД $(q, l(v))=1$, соотношений вида $(25)$ с $q \mid l_{1}(x, e)$ и с достаточно малым $d(x, e)$. Соотношения вида (19) с гладкими $s_{1}$ и $s_{2}$ мы будем стремиться получать в виде линейных комбинаций пар таких соотношений, найденных для какого-либо $q$, достаточно близкого к $p$.

При фиксированном $v$ и заданном $q, \mathrm{HOД}(q, l(v))=1$, определяем

$$
\gamma=\gamma(q)=\frac{\beta^{-1}-m}{q},
$$

где $\beta$ - число из (28), $m$ - число из (33). Так как $0<m(q)<q, 1<\beta^{-1}<2$, имеем $-1<\gamma(q)<0$. Для целых, взаимно простьх чисел $k<0$ и $e$ определяем

$$
\delta=\delta(q)=\gamma-\frac{k}{|e|} .
$$

Укажем сначала условия, обеспечивающие возможность $(x, e)$-замены с $q \mid l_{1}(x, e)$.

ПРЕДЛОЖЕНИЕ 8. Пусть $p$ - простое число и выполняются условия:

а) $v \in I_{1}-$ иелое число,

б) $q$ - натуральное число, НОД $(q, l(v))=1$ c $l(v)$ из (31),

в) $k<0$, - - иелые числа, не равные нулю,

г) $m(q)$ находится по (33), $x-$ no (34),

д) $\gamma(q)$ - число из (35) и для $\delta(q)$ из (36) выполнено

$$
|\delta|<\frac{r}{p q}
$$

сммалым иелым $r \ll p$.

Тогда

1) справедливь неравенства

$$
1-\frac{r}{p}<\frac{x}{\beta^{-1}|e|}<1+\frac{r}{p}
$$

2) справедливо неравенство

$$
x>|e|
$$

для любого $v$ при $\delta<0$ и почти для всех $v$, когда $\delta>0$ (если $r \leqslant 23$, то возмохни лишь два исключения - когда $v-3 p / 8<1$ и когда $2 p / 5-v<1$ ). 
СлЕДСтвиЕ. Если выполнены условия а)-Г), $|\delta|<1 /(p q) u q<23 p, m o(x, e)-з a-$ мена с соответствующими е и $x$, всегда приводит к соотношению (25) $c q \mid l_{1}(x, e)$ ( с единственным возможным исключением).

ТЕОРема 1. В условиях предложения 8 предположим дополнительно, что

$$
\frac{|e|}{q} \leqslant \nu
$$

где $\nu$ - малое челое число. Тогда в результате $(x, e)$-замены мы приходим к соотношению (25) $\mathrm{c} q \mid l_{1}(x, e)$, в котором

$$
\frac{l_{1}(x, e)}{q} \leqslant 13 \nu
$$

ДокАЗАТЕльСтво. Пусть $v \in I_{1}^{\prime}$. Тогда по правилу С берется $e>0$ и согласно (38)

$$
x<\beta^{-1} e\left(1+\frac{r}{p}\right)
$$

где $r \ll p$-малое целое число. Так как $\beta^{-1}<2$ (см. (29)), отсюда следует, что

$$
l_{1}(x, e)=5 x+3 e<13 e+10 r \frac{e}{p}
$$

или

$$
\frac{l_{1}(x, e)}{e}<13+\frac{10 r}{p}
$$

Отсюда следует, что

$$
\frac{l_{1}(x, e)}{q}<13 \frac{e}{q}+10 \frac{r e}{(p q)}
$$

и ввиду условия (39)

$$
\frac{l_{1}(x, e)}{q} \leqslant 13 \nu .
$$

Аналогично рассуждаем и в случае $v \in I_{1}^{\prime \prime}$. Теорема доказана.

Далее в качестве чисел $q$, участвующих в формулировках предложения 8 и теоремы 1 , будем использовать числа вида $q=p+q_{0}$, где $q_{0}$ - гладкое число или, по крайней мере, число из области с большой плотностью гладких чисел. Для таких $q$ мы рассматриваем $\gamma(q)$, точнее, его приближенное с точностью до $1 / 100 p^{2}-1 / 10000 p^{2}$ значение $\tilde{\gamma}$. Это $\tilde{\gamma}$ мы аппроксимируем числами $k /|e|$ по методу цепных дробей. Из теории цепных дробей следует, что нужное приближение достигается при значении знаменателя $|e|$ подходящей дроби, не превосходящего $\nu p$, где $\nu$ - малое целое число. Но для таких $q$ и $\delta=\gamma-k /|e|$ оказываются выполненньми условие д) предложения 8 и условие (39) теоремы 1 . Тем самым, мы в результате произведенной $(x, e)$-замены приходим к соотношению $(25)$ с $q \mid l_{1}(x, e)$ и $l_{1}(x, e) / q \leqslant 13 \nu$. Мы покажем ниже, что указанньй выбор чисел $q$ и уровня приближений к $\gamma(q)$ оказьвается благоприятным также и для получения искомых соотношений $(25)$ с относительно мальми $d(x, e)$.

Сначала преобразуем равенство (30) из предложения 6. 
ПРЕДЛОЖЕНИЕ 9. Пусть выполнены условия а)-д) предложсения 8. Тогда справедливо

$$
|d(x, e)|=\beta_{1}|\delta||e| q p,
$$

$2 \partial e$

$$
\beta_{1}(v)=\beta_{1}= \begin{cases}(2 \alpha-1)(2+\alpha)^{-1}, & v \in I_{1}^{\prime}, \\ (2-3 \alpha)(2+\alpha)^{-1}, & v \in I_{1}^{\prime \prime},\end{cases}
$$

$c \delta=\gamma-k /|e|, \gamma u з(35), \alpha u з(20)$.

Функиия $\beta_{1}(v)$ монотонно убавает в $I_{1}^{\prime}$ от $1 / 8$ до $1 / 13$ и монотонно возрастает в $I_{1}^{\prime \prime}$ om $1 / 13$ до $1 / 5$.

ДокАЗАТЕльСтво. Обозначим $\Delta=\delta|e|$. Из (36) и (35) получаем

$$
\gamma|e|=k+\Delta \quad \text { и } \quad \beta^{-1}|e|-|e| m=k q+\Delta q
$$

т.е.

$$
\beta^{-1}|e|=x+\Delta q
$$

Нетрудно убедиться, что из условия д) в предложении 8 вытекает, что $x+\Delta q \neq 0$. Поэтому

$$
\beta=\frac{|e|}{x+\Delta q}
$$

Отсюда получаем, что

$$
\beta-\frac{|e|}{x}=\frac{|e| \Delta q}{x(x+\Delta q)}=\frac{\beta \delta|e| q}{x} .
$$

Подставляя это выражение в (30), получаем

$$
|d(x, e)|=\beta|\delta||e| q(1-\alpha) v
$$

Учитьвая, что $v=(2+\alpha)^{-1} p$, и обозначая

$$
\beta_{1}(v)=\beta(1-\alpha)(2+\alpha)^{-1}
$$

приходим к (40).

Пусть теперь $v \in I_{1}$. Тогда

$$
\beta_{1}=\frac{2 \alpha-1}{2+\alpha}=\frac{2 p-5 v}{p} \quad \text { для } v \in I_{1}^{\prime}
$$

и

$$
\beta_{1}=\frac{8 v-3 p}{p} \quad \text { для } v \in I_{1}^{\prime \prime} .
$$

Отсюда следуют требуемые утверждения для $\beta_{1}$. Предложение доказано.

Подытожим наши рассмотрения. 
ТЕОРемА 2. Пусть выполнены условия предложсения 8. Если мы производим $(x, e)$-замену с соответствующими $е, x$, при которой $q \mid l_{1}(x, e) u$

а) $q=p+q_{0}$, где $q_{0}-$ малое челое число,

б) $\beta_{1}|e| \cdot|\delta|=(1+\lambda) / p$, где $\lambda-$ рациональное число со знаменателем, взаимно простым с $p$, такое, что $\lambda \approx r / p, r$-малое целое число,

то в результате этой замены приходим к соотношению

$$
\frac{l_{1}(x, e)}{q} q_{0} v \equiv d(x, e)(\bmod p)
$$

с малыми иелыми $l_{1} / q, q_{0}, d$.

ДокАЗАТЕльСтво. Применим предложение 9. По формуле (40), учитьвая а) и б), получаем

$$
|d(x, e)|=\frac{1}{p}(1+\lambda) q p=(1+\lambda)\left(p+q_{0}\right)=p+\lambda\left(p+q_{0}\right)+q_{0} .
$$

Отсюда следует, что $\lambda\left(p+q_{0}\right)$ - целое число. Его величина по условию примерно равна $r\left(p+q_{0}\right) / p=r+\left(q_{0} / p\right) r$, т.е. меньше, чем на 1 , отличается от малого целого $r$. Таким образом, $|d(x, e)|$ по модулю $p$ представляется как сумма малых целых чисел:

$$
|d(x, e)| \equiv \lambda\left(p+q_{0}\right)+q_{0}
$$

т.е. сравнимо с малым целым числом. Итак, в результате $(x, e)$-замены мы приходим к соотношению

$$
\frac{l_{1}}{q} q_{0} v \equiv d(\bmod p)
$$

с мальпи цельпи $l_{1} / q, q_{0}, d$. Теорема доказана.

Теоремы 1 и 2 показьвают, что получение с помошью $(x, e)$-замен соотношений $(19)$ с гладким $s_{1}$ практически обеспечивается, если брать $q=p+q_{0}$ с малым гладким $q_{0}$ и установить уровень приближения к $\gamma(q)$, гарантирующий его достижение подходящими дробями со знаменателями $|e|=\nu p$, где $\nu$ не превьшает малого целого числа. Покажем, что общий закон аптроксимации I (см. п. 1) гарантирует для каждого такого выбранного $q$ существование бесконечно многих пар $(|e|, k)$ целых чисел, для которых вьполнено также условие б) теоремы 2 с $\delta=\gamma(q)-k /|e|, \beta_{1}$ из (41).

ТЕОРемА 3. Пусть выполняются условия предложсения 8 и при этом берем число $q=p+q_{0}$, где $q_{0}$ - малое целое число (т.е. выполнено условие а) теоремы 2). Тогда существует бесконечно много пар иельх чисел $(|e|, k)$ со свойством

$$
\beta_{1}|e||\delta|=\frac{1}{p}(1+\lambda)
$$

где $\lambda \approx r / p$ смалым целым числом $r, \delta=\gamma(q)-k /|e|, \beta_{1}-$ число из (41) (т.е. выполнено условие б) теоремы 2). 
ДокАЗАТЕльСтво. Пусть $\lambda$ - положительное рациональное число. Рассмотрим неравенство

$$
\left|z \gamma-y-\frac{\lambda}{\beta_{1} p}\right|<\frac{1}{\beta_{1} p}
$$

с $\beta_{1}$ из (41).

Согласно первому из цитированных в начале п. 1 общих законов аппроксимации, неравенство (42) имеет бесконечно много решений в целых числах при $3 / z \leqslant 1 /\left(\beta_{1} p\right)$, или $3 \beta_{1} p \leqslant z$. Но мы рассматриваем такой уровень приближений к $\gamma(p)$, что знаменатели подходящих дробей при наилучшем приближении имеют величину вида $\nu p \mathrm{c} \nu>1$. Так как $\beta_{1}<1 / 5$ по предложению 9 , для значений $z$ из этой области условие $3 \beta_{1} p \leqslant z$ выполняется. Для любого целочисленного решения $(z, y)=(|e|, k)$ неравенства $(42)$ выполнено

$$
|| e\left|\gamma-k-\frac{\lambda}{\beta_{1} p}\right|<\frac{1}{\beta_{1} p}
$$

и пусть

$$
|e| \gamma-k-\frac{\lambda}{\beta_{1} p}<\frac{1}{\beta_{1} p}
$$

(в другом случае, когда $|e| \gamma-k-\lambda /\left(\beta_{1} p\right)>-1 /\left(\beta_{1} p\right)$ рассуждаем аналогично). Тогда

$$
|e| \gamma-k-\frac{1}{\beta_{1} p}<\frac{\lambda}{\beta_{1} p} \quad \text { и } \quad|e| \gamma-k-\frac{1}{\beta_{1} p}=\frac{\lambda_{1}}{\beta_{1} p}
$$

с рациональным $\lambda_{1}, \lambda_{1} / \lambda<1$. Следовательно,

$$
\beta_{1}|e||\delta|=\frac{1}{p}+\frac{\lambda_{1}}{p} .
$$

Полагая $\lambda_{1} \approx r / p$ с малым цельг $r$, приходим к утверждению теоремы.

Отметим также, что общий закон апроксимации II, цитированный в п. 1, гарантирует существование решения любого сравнения вида (42) при значениях $z=|e| \leqslant C \beta_{1} p$, где $C$ - некоторое положительное число.

Итак, действуя по изложенному вьше методу (см. предложение 8), нам при данном $q$ нужно искать такие приближения $k /|e| \mathrm{k} \gamma(q)$, чтобы для $|e|, \delta=\gamma-k /|e|$ удовлетворялось условие б) теоремы 2. Возможный способ нахождения таких приближений дают результаты п. 1 (предложения $3,3^{\prime}$ и $4,4^{\prime}$ ). В них бесконечное множество достаточно хороших приближений к числу $\gamma$ получается из двух данных последовательных приближений в результате построения их всевозможных линейных комбинаций. Если эти исходные приближения приводят к соотношениям $\lambda_{1}\left(q_{0} v\right) \equiv d_{1}, \lambda_{2}\left(q_{0} v\right) \equiv d_{2}(\bmod p)$, где $\lambda_{i}=l_{1}\left(x_{i}, e_{i}\right) / q$, то для любых целых $s, t$

$$
\left(\lambda_{1} s+\lambda_{2} t\right)\left(q_{0} v\right) \equiv \lambda_{1} d_{1}+\lambda_{2} d_{2}(\bmod p) .
$$

Если при этом мы приходим к гладкому $\lambda_{1} d_{1}+\lambda_{2} d_{2}$, то, разумеется, $\lambda_{1} s+\lambda_{2} t$ не обязательно гладкое. Однако принятьй метод обеспечивает получение исходных соотношений с весьма малыми $\lambda_{1}$ и $\lambda_{2}$ (см. теорему 1 ). Поэтому значения линейных комбинаций $\lambda_{1} s+\lambda_{2} t$ часто остаются в области с высокой плотностью гладких чисел и сами оказьваются гладкими. На этих соображениях основывается вероятностный алгоритм получения рассматриваемых в этом пункте представлений, которьй мы дадим в п. 3. 
3. Формулировка алгоритма представления. Примеры и численные результаты. Пусть $p$ - большое (с несколькими десятками знаков в десятичном представлении) простое, $v$ - целое число, $0<v<p$. Напомним, что в п. 2 мы (предложение 5) свели задачу к случаю $v \in I=\left(\frac{3}{8} p, \frac{3}{7} p\right)$. Затем мы рассматривали лишь случай $v \in I_{1}=\left(\frac{3}{8} p, \frac{2}{5} p\right]$ отметив, что при $v \in\left(\frac{2}{5} p, \frac{3}{7} p\right)$ все рассуждения проводятся совершенно аналогично, с изменением, главным образом, вида некоторых формул. Здесь в целях экономии места мы сосредоточимся на случае $v \in I_{1}^{\prime}=\left(\frac{3}{8} p, \frac{5}{13} p\right]$; для $v \in\left(\frac{5}{13} p, \frac{2}{5} p\right]$ все действия по алгоритму производятся совершенно так же, все вьводы и результаты сохраняются.

Итак, пусть $v \in\left(\frac{3}{8} p, \frac{5}{13} p\right]$. Ищем пары гладких целых чисел $(\lambda, d)$, для которых

$$
\lambda v \equiv d(\bmod p)
$$

Разобьем алгоритм на несколько шагов.

Шаг 0 (выбор базы). Берем “базу" $\mathscr{B}$ - множество всех наименьших простьх чисел $\{2,3, \ldots\}$ в количестве $|\mathscr{B}|=c\left\lceil\log _{2} p\right\rceil$. (В наших экспериментах было $c=5$ ).

Шаг 1. Вычисляем (см. (20) и (28)) $\alpha(v)=(p-2 v) / v, \beta^{-1}(v)=(1-\alpha) /(2 \alpha-1)$ с точностью до $1 / 100 p^{2}$.

Шаг 2. Вводим переменньй параметр $q$. При каждом возврашении к шагу 2 берем следующее (не делящееся на 5 , иначе - пропускаем) число в таблище

$$
\begin{aligned}
& p \pm 1, p \pm 11, p \pm 21, \ldots \\
& p \pm 2, p \pm 12, p \pm 22, \ldots \\
& \ldots \ldots \ldots \ldots \ldots \ldots \ldots \\
& p \pm 10, p \pm 20, p \pm 30, \ldots
\end{aligned}
$$

последнее число в каждой строке не превьшает $p+5 P$ по абсолютной величине, где $P-$ максимальное число в $\mathscr{B}$.

Шаг 3. а) Находим целое число $\mu=\mu(q)$ (см. (32)) по таблице

$$
\mu=\left\{\begin{array}{l}
1, \text { если } q \equiv 3(\bmod 5), \\
2, \text { если } q \equiv 4(\bmod 5), \\
3, \text { если } q \equiv 1(\bmod 5), \\
4, \quad \text { если } q \equiv 2(\bmod 5),
\end{array}\right.
$$

а затем $m(q)($ см. (33)):

$$
m=\frac{\mu(q) q-3}{5}
$$

б) Находим число $\gamma=\left(\beta^{-1}-m\right) / q$ с точностью до $1 /\left(10000 p^{2}\right)$. Это приближенное значение обозначаем $\tilde{\gamma}$.

Шаг 4. Строим последовательность приближений к $|\tilde{\gamma}|$ методом цепных дробей (см. п. 1), т.е. последовательность

$$
\widetilde{R} \equiv\left(\frac{k_{0}}{e_{0}}, \frac{k_{1}}{e_{1}}, \cdots, \frac{k_{f}}{e_{f}}=|\tilde{\gamma}|\right)
$$


состоящую из конечного числа (см. свойство 3) п. 1) несократимых рациональных дробей ( “подходящих дробей” для $|\tilde{\gamma}|$ ). Длина $f$ последовательности $\widetilde{R}$ оценивается в среднем как $3 \log p$.

В итоге получаем определяющие искомые приближения три целочисленные последовательности:

$$
\begin{gathered}
A=\left(a_{0}, a_{1}, a_{2}, \ldots, a_{f}\right), \quad K=\left(k_{0}, k_{1}, k_{2}, \ldots, k_{f}\right), \\
E=\left(e_{0}, e_{1}, e_{2}, \ldots, e_{f}\right) .
\end{gathered}
$$

Шаг 5 (нахождение множества $L D$ ). Обозначаем $E_{0}=\{e \in E, e \in(p / 10,12 p)\}$, $R_{0}=\left\{k / e \in \widetilde{R}, e \in E_{0}\right\}$. Для каждой дроби $k / e \in R_{0}$ находим

a) $x=e m-k q$ (см. (34)),

б) $l_{1}(x, e)=5 x+3 e($ см. $(23))$,

в) целое (см. предложение 8) $l_{1}(x, e) / q$,

г) $l_{2}(x, e)=2 x+e($ см. $(24))$,

д) $l_{1}(x, e) v / q$,

е) $l_{2}(x, e)-l_{1}(x, e) v / q$

и, наконец,

$$
d(x, e)=\frac{l_{1}(x, e)}{q}(q-p) v-\left(l_{2}(x, e)-\frac{l_{1}(x, e)}{q} v\right) p .
$$

Обозначаем $\lambda=\lambda(x, e)=l_{1}(x, e) / q$,

$$
L D=\left\{(\lambda(x, e), d(x, e)): \frac{k}{e} \in R_{0}\right\}
$$

Для $\lambda(x, e), d(x, e)$ справедливо $\lambda(x, e) q_{0} v \equiv d(x, e)(\bmod p)$, где $q_{0}=q-p$.

Шаг 6. Берем две (последовательные) пары чисел $\left(\lambda_{1}, d_{1}\right)$ и $\left(\lambda_{2}, d_{2}\right)$ из $L D$ с наименьшими по абсолютной величине $d_{1}, d_{2}$. Для них образуем все возможные пары

$$
(\lambda, d)=\left(s \lambda_{1}+t \lambda_{2}, s d_{1}+t d_{2}\right),
$$

с целыми числами $s>0, t, s \leqslant\left|c_{1}\right|,|t| \leqslant c_{2}$ (в примерах ниже $c_{1}=c_{2}$ и $c_{1}=750$ для $p$ порядка $10^{16}, c_{1}=1000$ для $p$ порядка $10^{21}, c_{1}=1500$ для $p$ порядка $\left.10^{31}\right)$. Отбираем те пары, в которых как $\lambda$, так и $d$ являются гладкими (т.е. делятся лишь на произведения чисел из $\mathscr{B}$ ). Если общее количество таких неколлинеарных пар (полученных к данному времени) меньше $2|\mathscr{B}|$, то переходим к шагу 2 и берем следующее значение параметра $q$. Работа алгоритма прекращается тогда, когда либо набрано $2|\mathscr{B}|$ пар $(\lambda, d)$ с гладкими $\lambda$ и $d$ (т.е. фактически $2|\mathscr{B}|$ соотношений (19) с гладкими $s_{1}=\lambda$ и $s_{2}=d$ ), либо будут исчерпаны все значения $q$.

Для выяснения степени эффективности представленного алгоритма и поиска возможных путей ее повышения были проведены на ЭВМ некоторые эксперименты. Подчеркнем, что они не имели достаточно систематического характера, а их целью было, прежде всего, стремление проиллюстрировать развитыев работе теоретические рассмотрения. Были взяты три групшы значений простого числа $p$ : a) порядка $10^{15}-10^{16}$, б) порядка $10^{20}-10^{21}$, в) порядка $10^{30}-10^{31}$. Более или менее подробно рассматривались лишь случаи а) и б). 
а) В результате экспериментов с числами $p$ порядков $10^{15}-10^{16}$ получалось, что современньй компютер, действуя, по описанному алгоритму, при базе из 250 чисел (максимальное число в базе - 1583) находим 1000 независимых (т.е. неколлинеарных) соотношений (19)

$$
s_{1} v \equiv s_{2}(\bmod p)
$$

с гладкими $s_{1}, s_{2}$ за время менее 20 минут. За это время “пробегается" $1 \%$ значений $q$; искомые соотношения обнаруживаются практически при любом взятом $q$ (в $100 \%$ или иногда чуть в меньшем, $>90 \%$ случаев).

Приведем примеры.

Рассмотрим $p=1470002300000951, v=554318181250360\left(v \in\left(\frac{3}{8} p, \frac{5}{13} p\right)\right)$.

Шаг 0. Берем базу $\mathscr{B}=\{2,3, \ldots, 1583\}$ из $250 \approx 5\left\lceil\log _{2} p\right\rceil$ наименьших простых чисел, $P=1583$.

Шаг 1. Вычисляем $\alpha(v)$ (см. (20)) и $\beta^{-1}(v)($ см. $(28))$.

Шаг 2. Берем $q=p+1: q=1470002300000952$.

Шаг 3. Находим $\mu(q)$ (см. (32)), $m(q)$ (см. (33)) и $\gamma(q)$ (см. (35)), беря затем его приближенное значение $\tilde{\gamma}$ :

$$
\tilde{\gamma}=-0.3999999999999994062240559346477321
$$

(34 знака после запятой).

Шаг 4. Методом цепных дробей, как описано в п. 1 , строим последовательности $A$, $K, E, k_{i} / e_{i} \approx|\tilde{\gamma}|, i=1,2, \ldots, f, k_{i} \in K, e_{i} \in E$. При этом $f=51$.

Шаг 5. Находим, множество $L D$ пар $(\lambda, d)$, т.е. фактически следующие 7 соотношений вида $(25)$ с $V=(q-p) v$ :
1) $V \equiv 554318181250360(\bmod p)$,
2) $2 V \equiv-361365937500231(\bmod p)$,
3) $3 V \equiv 192952243750129(\bmod p)$,
4) $5 \mathrm{~V} \equiv-168413693750102(\bmod p)$,
5) $8 V \equiv 24538550000027(\bmod p)$,
6) $53 \mathrm{~V} \equiv-21182393749940(\bmod p)$,
7) $61 \mathrm{~V} \equiv 3356156250087(\bmod p)$.

Шаг 6. Берем два последних соотношения $\lambda_{1} V \equiv d_{1}, \lambda_{2} V \equiv d_{2}$ с $\lambda_{1}=61, \lambda_{2}=53$, полученные на предыдущем шаге, и образуем всевозможные пары с $s>0:\left(\lambda_{1} s+\lambda_{2} t\right.$, $\left.d_{1} s+d_{2} t\right), s,|t| \leqslant 750$. Находим в результате 7 искомых соотношений $(25)$ с гладкими $s_{1}$ и $s_{2}$ :

1) $3 \times 3 \times 3 \times 7 \times 71 \mathrm{~V}=13419 \mathrm{~V} \equiv 184036193768780$

$$
=2 \times 2 \times 5 \times 37 \times 109 \times 139 \times 167 \times 227 \times 433(\bmod p)
$$

$(s=200, \quad t=+23)$,

2) $71 \times 313 \mathrm{~V}=22223 \mathrm{~V} \equiv-6332081219100$

$$
=2 \times 2 \times 3 \times 3 \times 5 \times 5 \times 7 \times 13 \times 293 \times 367 \times 719(\bmod p)
$$

$(s=320, \quad t=+51)$,

3) $5 \times 19 \times 379 V=36005 V \equiv 4888806300073=101 \times 191 \times 359 \times 727 \times 971(\bmod p)$ $(s=519, \quad t=+82)$, 
4) $47 \times 863 \mathrm{~V}=40561 \mathrm{~V} \equiv 14571181306415$

$$
=5 \times 19 \times 59 \times 59 \times 251 \times 349 \times 503(\bmod p)
$$

$(s=585, \quad t=+92)$,

5) $2 \times 73 \times 293 \mathrm{~V}=42778 \mathrm{~V} \equiv 16056212559499$

$$
=7 \times 19 \times 31 \times 53 \times 89 \times 661 \times 1249(\bmod p)
$$

$(s=617, \quad t=+97)$,

6) $71 \times 617 \mathrm{~V}=43807 \mathrm{~V} \equiv 48572318810951$

$$
=13 \times 13 \times 43 \times 43 \times 61 \times 67 \times 73 \times 521(\bmod p)
$$

$(s=633, \quad t=+98)$,

7) $89 \times 569 \mathrm{~V}=50641 \mathrm{~V} \equiv 63095881320464$

$=2 \times 2 \times 2 \times 2 \times 59 \times 293 \times 373 \times 599 \times 1021(\bmod p)$

$(s=732, \quad t=+113)$.

б) Для простых чисел $p$ порядка $10^{20}-10^{21}$ эксперименты проводились до момента получения нескольких соотношений (19) с гладкими $s_{1}$ и $s_{2}$. База, как и прежде, составляла $\approx 5\left\lceil\log _{2} p\right\rceil$ наименьших простых чисел $(|\mathscr{B}|=330, P=2213)$. В результате для таких $p$ 3-4 искомых соотношения находились за 11-28 минут работы компютера. Хотя такие соотношения в этих условиях получались не при каждом $q$, а в $10-20 \%$ случаев (испытывались лишь 13-31 значений $q$ ), среди них бывали несколько соотношений при одном и том же $q$, что особенно перспективно при последующем составлении и решении линейных систем уравнений над кольцом $\mathbb{Z}_{p-1}$, решая которыеи получаем $\lg v$.

Вот пример соотношения для $p$ порядка $10^{21}$.

Пусть $p=145000000003800000637, v=55022115386058654087$.

Берем $q=145000000003800000638$.

Исходя из получаемых в ходе работы алгоритма соотношений $(V=(q-p) v)$

$$
\begin{gathered}
\lambda_{1} V=29 V \equiv 641346153900961516 \equiv d_{1}(\bmod p), \\
\lambda_{2} V=-21 V \equiv 4535576923168269269 \equiv d_{2}(\bmod p),
\end{gathered}
$$

приходим к соотношению

$$
\begin{aligned}
\left(\lambda_{1} s+\lambda_{2} t\right) V & =3044 V=2 \times 2 \times 761 V \equiv 12319230773542305093 \\
& =3 \times 13 \times 67 \times 113 \times 139 \times 149 \times 607 \times 1553 \times 2137 \\
& \equiv d_{1} s+d_{2} t(\bmod p) \quad(\text { при } s=97, \quad t=11)
\end{aligned}
$$

в) Дляпростых чисел порядка $10^{30}-10^{31}$ рассматривались лишш единичныепримеры. В них бралась база $\mathscr{B}$ с $|\mathscr{B}|=500$ и $P=p_{\max }=3571$. Работа велась до получения первого соотношения (19) с гладкими $s_{1}$ и $s_{2}$. Такие соотношения получались после многочасовой (до полусуток) работы компьютера.

Приведем пример.

Пусть

$$
\begin{gathered}
p=1380000000076000000004200000847, \\
v=524734615413480769232364423398
\end{gathered}
$$


Берем $q=138000000076000000004200002358$.

Исходя из получаемых в ходе работы алгоритма соотношений $(V=(q-p) v)$

$$
\begin{aligned}
& \lambda_{1} V=-97 V \equiv 1626927920096154113803029491 \equiv d_{1}(\bmod p), \\
& \lambda_{2} V=-86 V \equiv 15669235063961538698878974609 \equiv d_{2}(\bmod p),
\end{aligned}
$$

приходим при $s=1259, t=-130$ к соотношению

$$
\begin{aligned}
& \left(s \lambda_{1}+t \lambda_{2}\right) V=-110943 V=-3 \times 3 \times 3 \times 7 \times 587 V \\
& \quad \equiv 11301693086057998423747429999 \equiv\left(s d_{1}+t d_{2}\right) \\
& \quad \equiv 11 \times 43 \times 47 \times 59 \times 61 \times 283 \times 359 \times 1009 \times 1447 \times 1483 \times 2269(\bmod p) .
\end{aligned}
$$

Мы видим, таким образом, что попытка ограничиться весьма маленькой базой делает в случае в) алгоритм менее эффективньм, чем в случаях а) и б).

Вообще, достаточно умеренное увеличение базы составляет один из резервов повышения эффективности алгоритма. Действительно, в этом случае ситуация приблизится к случаю а), когда практически для каждого из рассматриваемых значений $q$ мы получали несколько соотношений (19) с гладкими $s_{1}$ и $s_{2}$. Тем самым, скорее составится система линейных уравнений над кольцом $\mathbb{Z}_{p-1}$, из которой определится $\lg v$. Другим существенным резервом повышения эффективности алгоритма является в перспективе уход от чистого перебора в шаге 6.

Автор считает своим долгом выразить глубокую благодарность заведующему отделом “Дискретная математика" МИРАН А. М. Зубкову за чрезвычайно ценные советы и замечания. Автор благодарен сотрудникам лаборатории № 22 Института проблем управления РАН (руководитель - Д.В. Тюкавкин, ведущий научный сотрудник С. Э. Шаронов) за создание компютерной программы, реализующей изложенный в работе алгоритм, и проведение вычислительных экспериментов. Автор благодарит также Л.К. Мишину за большую помошь при проведении ручных экспериментов.

\section{СПИСОК ЦИТИРОВАННОЙ ЛИТЕРАТУРЫ}

[1] Боревич З. И., Шафаревич И. Р. Теория чисел. М.: Наука, 1964.

[2] Хинчин А. Я. Цепные дроби. М.: ОНТИ НКТП, 1935.

[3] Коблиц Н. Курс теории чисел и криптографии. М.: ТВП, 2001.

[4] Adleman L. M. A subexponential algorithm for discrete logarithm problem with applications to cryptography // Proc. of the 20th Annual IEEE Symposium on Foundations of Computer Science, 1979. P. 55-60.

[5] Coppersmith D., Odlyzko A., Schroeppel R. Discrete logarithms in $G F(p) / /$ Algorithmica. 1986. V. 1. P. 1-15.

[6] Schirokauer O. Discrete logarithms and local units // Proc. Trans. Roy. Sci. London Ser. A. 1993. V. 345. P. 409-423. 